Accepted by The Astrophysical Journal

\title{
Low Mass Companions for Five Solar-Type Stars from the Magellan Planet Search Program ${ }^{1}$
}

\author{
Dante Minniti ${ }^{2,6}$, R. Paul Butler ${ }^{3}$, Mercedes López-Morales ${ }^{3,7}$, Stephen A. Shectman ${ }^{4}$, Fred \\ C. Adams ${ }^{5}$, Pamela Arriagada ${ }^{2}$, Alan P. Boss ${ }^{3}$, John E. Chambers ${ }^{3}$ \\ dante@astro.puc.cl
}

\begin{abstract}
We report low mass companions orbiting five Solar-type stars that have emerged from the Magellan precision Doppler velocity survey, with minimum $(M \sin i)$ masses ranging from 1.2 to $25 \mathrm{M}_{\mathrm{JUP}}$. These nearby target stars range from mildly metal-poor to metal-rich, and appear to have low chromospheric activity. The companions to the brightest two of these stars have previously been reported from the CORALIE survey. Four of these companions (HD 48265-b, HD 143361-b, HD 28185-b, HD 111232-b) are low-mass Jupiter-like planets in eccentric intermediate and long-period orbits. On the other hand, the companion to HD 43848 appears to be a long period brown dwarf in a very eccentric orbit.
\end{abstract}

Subject headings: planetary systems - stars: individual (HD 48265, HD 143361, HD 43848, HD 28185, HD 111232)

\footnotetext{
${ }^{1}$ Based on observations obtained with the Magellan Telescopes, operated by the Carnegie Institution, Harvard University, University of Michigan, University of Arizona, and the Massachusetts Institute of Technology.

${ }^{2}$ Department of Astronomy, Pontificia Universidad Católica de Chile, Casilla 306, Santiago 22, Chile

${ }^{3}$ Department of Terrestrial Magnetism, Carnegie Institution of Washington, 5241 Broad Branch Road NW, Washington D.C. USA 20015-1305

${ }^{4}$ Carnegie Observatories, 813 Santa Barbara Street, Pasadena, CA USA 91101

${ }^{5}$ Astronomy Department, University of Michigan, Ann Arbor, MI USA 48109

${ }^{6}$ Specola Vaticana, V00120 Citta' del Vaticano, Italy

${ }^{7}$ Hubble Fellow
} 


\section{Introduction}

A decade after the milestone discoveries of the first extrasolar planet (Mayor \& Queloz 1995), the first planetary system (Butler et al. 1999), and the first transiting planet (Henry et al. 2000; Charbonneau et al. 2000) precision Doppler surveys remain the dominant method for finding extrasolar planets orbiting nearby stars. Most of the planets orbiting stars within 100pc have been found from precision velocity surveys. Current surveys now cover nearly all main sequence $\mathrm{G}$ and $\mathrm{K}$ stars within 50 pc. Northern hemisphere planet radial velocity surveys include the Lick 3-m, Elodie 1.93-m, Keck 10-m, and the HET 11-m. Surveys in the southern hemisphere include the HARPS 3.6-m, AAT 3.9-m, and the Coralie 1.2-m.

With the unexpected diversity of extrasolar planets found to date (Butler et al. 2006), every new planet continues to contribute to our understanding. Planets around the stars closer than 100 pc are especially valuable as these are the stars that will be prime targets for studies by future interferometry and direct imaging missions.

In this work we present the detection of low-mass companions to five nearby solar-type stars, including four of planetary mass, and one likely brown dwarf. The brightest two of the objects reported here have previously been detected by the CORALIE team (Santos et al. 2001; Mayor et al. 2004). These sub-stellar companions were found by the Magellan precision Doppler survey, which has been operated over the past 4 years, and is focused on late F, G, $\mathrm{K}$, and $\mathrm{M}$ dwarfs within $50 \mathrm{pc}$. This program is complementary to the Magellan N2K search for "Hot Jupiters" (Fischer et al. 2003; Lopez-Morales et al. 2008), which surveys metal rich stars primarily beyond $50 \mathrm{pc}$, with time allocated by the Chilean National TAC. The same hardware and software reduction packages are used by both programs.

This paper is organized as follows. Section 2 provides an overview of the Magellan Planet Search Program. Section 3 contains a summary of properties of the 5 stars, Doppler velocity measurements, and best-fit orbital solutions for companions. A discussion is given in Section 4.

\section{The Magellan Planet Search Program}

The Magellan Planet Search Program makes use of the MIKE echelle spectrograph (Bernstein et al. 2003) on the 6.5-m Magellan II (Clay) telescope. The resolution of these spectra is $\mathrm{R} \sim 50000$, spanning wavelengths from 3900-6200 $\AA$, divided into a blue and a red CCD. Wavelength calibration is carried out by means of an iodine absorption cell (Marcy \& Butler 1992) which superimposes a reference iodine spectrum directly on the stellar spectra. ThAr lamps are not used in any part of the reduction. The wavelength solution is derived 
directly from the Iodine spectrum, using the spectral "atlas" of this cell taken with the NIST FTS spectrometer at a resolution of 1,000,000 and a S/N of 1,000 as the metric (Butler et al. 1996; Valenti et al. 1995). The standard Iodine technique works between $5000 \AA$ and 6200 $\AA$. Below $5000 \AA$, Iodine molecules disassociate, so there are no lines. Above $6200 \AA$ Iodine lines are too weak to be useful, unless the Iodine cell is heated to several hundred degrees C. Thus, only the spectral orders of the MIKE red CCD are used to measure the radial velocities, while the blue orders are used to monitor the activity of the CaII $\mathrm{H}$ and $\mathrm{K}$ lines.

We have monitored a number of stable main sequence stars of spectral types ranging from late F to mid K. Several example of these RV stable stars are shown in Figures 1 and 2. This system currently achieves measurement precision of $5 \mathrm{~m} \mathrm{~s}^{-1}$, as demonstrated by these figures.

The Magellan Precision Doppler Survey began in December 2004, and is currently surveying about 400 main sequence dwarfs ranging in spectral type from late $\mathrm{F}$ to mid-M. The spectrum of stars earlier than F7 do not contain enough Doppler information to achieve precision of $5 \mathrm{~m} \mathrm{~s}^{-1}$, while stars later than M5 are too faint even for a 6.5-m telescope. Magellan/MIKE can reach $5 \mathrm{~m} / \mathrm{s}$ for stars as faint at $\mathrm{V}=9$ with 10 minute exposures.. The stars in the Magellan program have been chosen to minimize overlap with the AAT 3.9-m and Keck 10-m surveys. Subgiants have not been removed. Stellar jitter for subgiants is small, $\lesssim 5 \mathrm{~m} / \mathrm{s}$. Stars more than 2 magnitudes above the main sequence have much larger jitter, thus have been removed from the observing list based on Hipparcos distances (Perryman et al. 1997, ESA 1997).

Stars with known stellar companions within 2 arcsec are also removed from the observing list as it is operationally difficult to get an uncontaminated spectrum of a star with a nearby companion. Otherwise there is no bias against observing multiple stars. The Magellan target stars also contain no bias against brown dwarf companions.

\section{Companions from the Magellan Survey}

This paper reports on four planet-mass candidates and a brown dwarf candidate that have emerged from the Magellan survey. The stellar properties of the five host stars are given in Table 1. The first two columns provide the HD catalog number and the Hipparcos catalog number respectively. Spectral types are from a calibration of $B-V$ and Hipparcos derived absolute magnitudes. The stellar masses are estimated by interpolation of evolutionary tracks (Fuhrmann 1998, Fuhrmann et al. 1997). The $[\mathrm{Fe} / \mathrm{H}]$ values listed in Table 1 are drawn from a variety of sources (given below). 
Figure 3 shows the $\mathrm{K}$ line for the 5 stars reported in this paper, in ascending order of B-V. The Sun (bottom) is shown for comparison. Mt. Wilson S values (Baliunas et al. 1995) have been measured from the $\mathrm{H} \& \mathrm{~K}$ lines in the Magellan spectra. The $\log \left(\mathrm{R}_{\mathrm{HK}}^{\prime}\right)$ values are listed in Table 1. These stars are all chromospherically quiet. Therefore, we can constrain the expected photosperic radial velocity jitter $(<3 \mathrm{~m} / \mathrm{s})$, and reject activity as the cause of the observed radial velocity variations of these stars discussed below.

The orbital parameters were obtained by fitting single planet Keplerian orbits to the radial-velocity data (Lopez-Morales et al. 2008). The orbital parameters of the five companions are listed in Table 2, while the individual Magellan Doppler velocity measurements are listed in Tables 3 through 7 . The properties of the host stars and of their companions are discussed in turn below.

\subsection{HD 111232}

HD 111232 is a G5V dwarf with $V=7.59$ and $B-V=0.701$. The Hipparcos parallax (Perryman et al. 1997) gives a distance of $29.41 \mathrm{pc}$ and an absolute visual magnitude $M_{V}=5.28$. The star is chromospherically quiet, with $\log \left(\mathrm{R}_{\mathrm{HK}}^{\prime}\right)=-5.13$. Its metallicity is $[\mathrm{Fe} / \mathrm{H}]=-0.36$ (Nordstrom et al. 2004).

Based on 38 observations, the CORALIE team (Mayor et al. 2004) discovered a planet orbiting HD 111232 with an orbital period $P=1143$ d, a radial velocity semi-amplitude of $K=159 / \mathrm{ms}$, and an eccentricity $e=0.20$. The RMS to the best-fit Keplerian orbit is 7.5 $\mathrm{m} \mathrm{s}^{-1}$.

Fifteen Magellan Doppler velocity observations of HD 111232 spanning 3.6 years have been made, as shown in Figure 4 and listed in Table 3. The observations span a full orbital period. The period of the best-fit Keplerian orbit is $P=1118 \pm 30$ days, the semi-amplitude is $K=162 \mathrm{~m} \mathrm{~s}^{-1}$, and the eccentricity is $e=0.19 \pm 0.10$, in agreement with the CORALIE results. The RMS of the velocity residuals to the Keplerian fit is $4.70 \mathrm{~m} \mathrm{~s}^{-1}$. The reduced $\chi_{\nu}$ of the Keplerian fit is 1.48. Given the mass of the star $\mathrm{M}_{\odot}=1.06$, the minimum mass of the companion is $M \sin i=8.1 \mathrm{M}_{J u p}$, and the orbital semi-major axis is $2.1 \mathrm{AU}$.

\subsection{HD 48265}

HD 48265 is a G5 IV/V star with $V=8.05$ and $B-V=0.747$. The Hipparcos parallax (Perryman et al. 1997) gives a distance of $87.4 \mathrm{pc}$ and an absolute visual magnitude, $M_{V}=3.34$. HD 48265 is chromospherically quiet, with $\log \left(\mathrm{R}_{\mathrm{HK}}^{\prime}\right)=-4.93$. Its metallicity is 
$[\mathrm{Fe} / \mathrm{H}]=0.17$ (Nordstrom et al. 2004).

Seventeen Magellan Doppler velocity observations have been made of HD 48265 spanning 4.4 years, as shown in Figure 5 and listed in Table 4. The observations span 2 orbital periods. The semi-amplitude of the best-fit Keplerian orbit is $K=29 \mathrm{~m} \mathrm{~s}^{-1}$, the period is $P=762$ days and the eccentricity is $e=0.24$. The RMS of the velocity residuals to the Keplerian orbital fit is $5.14 \mathrm{~m} \mathrm{~s}^{-1}$. The reduced $\chi_{\nu}$ of the Keplerian orbital fit is 1.37. Assuming a stellar mass of $\mathrm{M}=0.93 \mathrm{M}_{\odot}$ we derive a minimum mass of $M \sin i=1.3 M_{J u p}$ and an orbital semi-major axis of $1.3 \mathrm{AU}$.

\subsection{HD 28185}

HD 28185 is a G0 V star with $V=7.80$ and $B-V=0.750$. The Hipparcos parallax (Perryman et al. 1997) gives a distance of $39.6 \mathrm{pc}$ and an absolute visual magnitude, $M_{V}=$ 4.81. HD 28185 is chromospherically quiet with $\log \left(\mathrm{R}_{\mathrm{HK}}^{\prime}\right)=-4.81$. Its metallicity is $[\mathrm{Fe} / \mathrm{H}]=$ 0.24 (Fischer et al. 2005).

The planet orbiting HD 28185 was discovered by the CORALIE extra-solar planet search (Santos et al. 2001), using 40 high-precision radial velocity measurements over a 2-year period. The best-fit Keplerian solution to the CORALIE data has an RMS of $10 \mathrm{~m} \mathrm{~s}^{-1}$, and yields a period $(P)$ of 383 days, a semi-amplitude $(K)$ of $161 \mathrm{~m} \mathrm{~s}^{-1}$, and an eccentricity $e=0.07$. They marginally detect a long term linear drift suggesting the presence of a second long period companion.

Fifteen Magellan Doppler velocity observations have been made of HD 28185 over 3.6 years, as shown in Figure 6 and listed in Table 5. These observations span three orbital periods. The best-fit Keplerian orbit to the Magellan data yields a period $P=379 \pm 2$ days, a semi-amplitude $(K)$ of $163 \mathrm{~m} \mathrm{~s}^{-1}$, and an eccentricity $e=0.05 \pm 0.03$, in agreement with the CORALIE results. The RMS of the velocity residuals to the Keplerian orbital fit is $7.33 \mathrm{~m} \mathrm{~s}^{-1}$. The reduced $\chi_{\nu}$ of the Keplerian orbital fit is 1.51 . Given the stellar mass $\mathrm{M}_{\odot}=1.24$, the minimum mass of the planet is $M \sin i=6.7 M_{J u p}$ with an orbital semi-major axis of $1.1 \mathrm{AU}$.

The best-fit Keplerian orbit to the Magellan data does not include a linear trend. The

largest term trend consistent with the Magellan data is $5 \mathrm{~m} \mathrm{~s}^{-1}$ per year. More observations will be needed to constraint the possible long term trend reported by the CORALIE team. 


\subsection{HD 143361}

HD 143361 is a G0 V star with $V=9.20$ and $B-V=0.773$. The Hipparcos parallax (Perryman et al. 1997) gives a distance of $59.35 \mathrm{pc}$ and an absolute visual magnitude, $M_{V}=5.33$. HD 143361 is chromospherically quiescent with $\log \left(\mathrm{R}_{\mathrm{HK}}^{\prime}\right)=-4.92$. Its metallicity is $[\mathrm{Fe} / \mathrm{H}]=0.29$ (Nordstrom et al. 2004). We note that this star has similar $B-V$ color and metallicity as HD 28185, but that they differ more than $20 \%$ in mass and about $0.5 \mathrm{mag}$ in absolute luminosity.

In our survey, twelve Magellan Doppler velocity observations have been made of HD 143361 over 4.49 years, as shown in Figure 7 and listed in Table 6. The observations span more than one orbital period. The semi-amplitude of the best-fit Keplerian orbit is $K=63$

$\mathrm{m} \mathrm{s}^{-1}$, the orbital period is $P=1086$ days and the eccentricity is $e=0.18$. The RMS of the velocity residuals to the Keplerian orbital fit is $3.88 \mathrm{~m} \mathrm{~s}^{-1}$. The reduced $\chi_{\nu}^{2}$ of the Keplerian orbital fit is 1.18 . Given the stellar mass $\mathrm{M}=1.00 \mathrm{M}_{\odot}$, the derived minimum mass of the companion is $M \sin i=3.1 M_{\text {Jup }}$ and the semimajor axis is $2.1 \mathrm{AU}$.

\subsection{HD 43848}

HD 43848 is a G2 V star with $V=8.65$ and $B-V=0.927$. The Hipparcos (Perryman et al. 1997) derived distance is $37.05 \mathrm{pc}$ and the absolute visual magnitude is $M_{V}=5.80$. The star is chromospherically quiet, with $\log \left(\mathrm{R}_{\mathrm{HK}}^{\prime}\right)=-4.97$. The reemission seen in Figure 3 is negligible, and consistent with the low activity index for this star, and it has no impact on the radial velocity signal interpretation. Its metallicity is $[\mathrm{Fe} / \mathrm{H}]=-0.03$ (Prieto \& Lambert 1999).

Ten Magellan Doppler velocity observations have been made of HD 43848 over 4.1 years, as shown in Figure 8 and listed in Table 7. The semi-amplitude of the best-fit Keplerian orbit is $K=544 \mathrm{~m} \mathrm{~s}^{-1}$, the period is $P=2371$ days and the eccentricity is $e=0.69$. The RMS of the velocity residuals to the Keplerian orbital fit is $7.16 \mathrm{~m} \mathrm{~s}^{-1}$. The reduced $\chi_{\nu}$ of the Keplerian orbital fit is 1.77 . Given the stellar mass $\mathrm{M}_{\odot}=0.93$, the derived minimum mass is $M \sin i=25 M_{J u p}$ with an orbital semi-major axis of $3.4 \mathrm{AU}$. This is likely a brown dwarf, unless the orbital inclination is smaller than about 18 degrees, in which case it could be a low mass M-dwarf. Nevertheless, such inclination is improbable (less than 5\%).

A low mass companion of spectral type M3.5-M6.5 for this star was previously discovered by Eggenberger et al. (2007) using astrometric measurements with VLT/NACO. Their estimated companion's mass gives $\mathrm{M}=0.14 \mathrm{M}_{\odot}$ with a separation of 30.9 AU. The time span of the Magellan observations is too short to detect the presence of this long period M dwarf. 
Because of its high eccentricity $(e=0.69)$, the companion for HD 43848 would have a maximum separation from the primary of nearly $6 \mathrm{AU}$ at apastron. At a distance of $37 \mathrm{pc}$, this yields an angular separation of 0.16 arcsec. We estimate a magnitude of $K \sim 6.6$ for the primary, but the magnitude of the brown dwarf companion is more uncertain. A dusty brown dwarf would be too faint, while for a clear brown dwarf we expect an absolute magnitude of $M_{K} \sim 10$, and apparent magnitude $K \sim 13$. The detection of this companion in the near-IR seems to be at the limit using current instrumentation at HST or VLT. However, this would be a prime target for detection with new instrumentation such as PRIMA at the VLTI.

We have also checked the dispersion in the Hipparcos measurements for this star in comparison with stars of similar magnitudes located at similar distances. However, the errors are comparable, and there is no evidence for higher dispersion that would indicate the presence of a massive companion, and at the same time the data are not good enough to secure the substellar nature of the companion.

\section{Discussion}

A new radial velocity search for extrasolar planets carried out at the Magellan Clay telescope with MIKE is presented. We demonstrate long term precision of $5 \mathrm{~m} / \mathrm{s}$ (Figures 1 and 2).

This paper also reports the detection of four planet mass companions and one prospective brown dwarf candidate orbiting nearby $\mathrm{G}$ dwarfs. Two of the planets belonging to the brightest two candidates of the present sample were initially discovered by the CORALIE team. These stars range from mildly metal poor to metal rich.

Figure 9 shown the eccentricity versus period for the known planets, highlighting the five objects studied here. The planet mass companions have circular to mildly eccentric orbits with periods ranging from 1 to 3 years. Note the extreme location of the candidate brown dwarf HD 43848-b, with high mass and high eccentricity. This figure suggests that objects like this might not be uncommon at large periods. Also, this object would be a prime target for detection with the future interferometric facilities.

Figure 10 shows the position of known extrasolar planetary system in the star mass vs apparent magnitude diagram. The new planets from our survey (this work plus LopezMoralez et al. 2008) are shown with large full circles. These first Magellan discoveries are among the faintest targets surveyed for planets, probing deeper in the Solar Neighborhood. Note also the group of low mass stars, a range that is also accessible by Magellan. We expect that the Magellan telescopes will keep on contributing to the discovery of southern extrasolar 
planets.

The Magellan Planet Search Program has designed and built a custom Planet Finder Spectrograph (Crane et al. 2006; Crane et al. 2008) for the Clay Telescope. This new instrument will be installed at the end of 2008. Advantages of this new spectrograph include higher throughput, higher resolution, active and passive temperature stabilization, fixed format, and all optics optimized for the Iodine region (5000 to 6200 Angstroms). The goal is to reach precision of $1 \mathrm{~m} \mathrm{~s}^{-1}$.

The Magellan Planet Search Program is also collaborating with Greg Henry and the Tennessee State University Automated Astronomy Group to install two dedicated 0.8-m robotic photometry telescopes at Las Campanas to monitor nearby southern hemisphere planet search stars with a precision of 1 milli-mag. We expect these telescopes will be operational in early 2009.

We are thankful to Dr. Andrea Richichi (ESO), and to Dr. John Debes (DTM) for useful comments about the possible detection of HD 43848-b. We are grateful to the NIST atomic spectroscopy staff, in particular to Dr. Gillian Nave and Dr. Craig Sansonetti, for their expert assistance in calibrating our Iodine cell with the NIST FTS RPB gratefully acknowledges support from NASA OSS grant NNX07AR4OG. MLM acknowledges support provided by NASA through Hubble Fellowship grant HF-01210.01-A awarded by the STScI, which is operated by the AURA, Inc., for NASA, under contract NAS5-26555. DM and PA are supported by the Basal CATA PFB 06/09, and FONDAP Center for Astrophysics 15010003. This paper has made use of the Simbad and NASA ADS data bases.

\section{REFERENCES}

Baliunas, S. L., Donahue, R. A., Soon, W. H., Horne, J. H., Frazer, J., Woodard-Eklund, L., Bradford, M., Rao, L. M., Wilson, O. C., Zhang, Q., Bennett, W., Briggs, J., Carroll, S. M., Duncan, D. K., Figueroa, D., Lanning, H. H., Misch, A., Mueller, J., Noyes, R. W., Poppe, D., Porter, A. C., Robinson, C. R., Russell, J., Shelton, J. C., Soyumer, T. Vaughan, A. H., \& Whitney, J. H. 1995, ApJ, 438, 269.

Bernstein, R., et al. 2003, SPIE, 4841, 1694

Butler, R. P., Marcy, G. W., Williams, E., McCarthy, C., Dosanjh, P., \& Vogt, S. S. 1996, PASP, 108, 500

Butler, R. P., Marcy, G. W., Fischer, D. A., Brown, T. M., Contos, A. R., Korzennik, S. G., Nisenson, P. \& Noyes, R. W. 1999, ApJ, 526, 916. 
Butler, R. P. and Wright, J. T. and Marcy, G. W. and Fischer, D. A., Vogt, S. S. and Tinney, C. G., Jones, H. R. A. Carter, B. D., Johnson, J. A., McCarthy, C. \& Penny, A. J., 2006, ApJ, 646, 505.

Charbonneau, D., Brown, T. M., Latham, D. W. \& Mayor, M. 2000, ApJ, 529, L49.

Crane, J., Shectman, S. A., \& Butler, R. P. 2006, SPIE, 6269, 96

Crane, J., et al. 2008, SPIE, in press

Eggenberger, A., et al. 2007, A\&A, 474, 273

ESA 1997, The Hipparcos and Tycho Catalogues (ESA SP-1200).

Fischer, D. A., et al. 2003, ApJ, 620, 481

Fischer, D. A., et al. 2005, ApJ, 622, 1102

Fuhrmann, K. 1998, A\&A, 338, 161.

Fuhrmann, K., Pfeiffer, M.J. \& Bernkopf, J. 1997, A\&A, 326, 1081.

Henry, G. W., Marcy, G. W., Butler, R. P. \& Vogt, S. S. 2000, ApJ, 529, L45.

Lopez-Morales, M., et al. 2008, AJ, submitted

Marcy, G. W. \& Butler, R. P. 1992, PASP, 104, 270.

Mayor, M., \& Queloz, D. 1995, Nature, 378, 355

Mayor, M., et al. 2004, A\&A, 415, 391

Nordstrom, B., et al. 2004, A\&A, 418, 989.

Perryman, M. A. C., et al. 1996, A\&A, 310, L21.

Perryman, M. A. C., et al. 1997, A\&A, 323, L49. The Hipparcos Catalog

Pourbaix, D., \& Arenou, F. 2001, A\&A, 372, 935.

Prieto, C. A. \& Lambert, D. L. 1999, A\&A, 352, 555.

Santos, N. C., et al. 2001, A\&A, 379, 999

Valenti, J., Butler, R. P. \& Marcy, G. W. 1995, PASP, 107, 966. 
Table 1. Stellar Properties

\begin{tabular}{|c|c|c|c|c|c|c|c|c|}
\hline $\begin{array}{l}\text { Star } \\
(\mathrm{HD})\end{array}$ & $\begin{array}{c}\text { Star } \\
\text { (Hipp) }\end{array}$ & $\begin{array}{l}\text { Spec } \\
\text { type }\end{array}$ & $\begin{array}{l}\mathrm{M}_{\text {Star }} \\
\left(\mathrm{M}_{\odot}\right)\end{array}$ & $\begin{array}{c}\mathrm{V} \\
(\mathrm{mag})\end{array}$ & $\mathrm{B}-\mathrm{V}$ & $\log \left(\mathrm{R}_{\mathrm{HK}}^{\prime}\right)$ & {$[\mathrm{Fe} / \mathrm{H}]$} & $\begin{array}{c}\mathrm{d} \\
(\mathrm{pc})\end{array}$ \\
\hline 111232 & 62534 & G5 V & 0.75 & 7.59 & 0.701 & -5.13 & -0.36 & 28.9 \\
\hline 48265 & 31895 & G5 V & 0.93 & 8.05 & 0.747 & -4.93 & 0.17 & 87.41 \\
\hline 28185 & 20723 & G0 V & 1.24 & 7.80 & 0.750 & -4.81 & 0.24 & 39.56 \\
\hline 143361 & 78521 & G0 V & 0.95 & 9.20 & 0.773 & -4.92 & 0.29 & 59.35 \\
\hline 43848 & 29804 & G2 V & 0.89 & 8.65 & 0.927 & -4.97 & -0.03 & 37.05 \\
\hline
\end{tabular}


Table 2. Orbital Parameters

\begin{tabular}{|c|c|c|c|c|c|c|c|c|c|}
\hline $\begin{array}{l}\text { Star } \\
(\mathrm{HD})\end{array}$ & $\begin{array}{l}\text { Period } \\
\text { (days) }\end{array}$ & $\begin{array}{c}K \\
\left(\mathrm{~m} \mathrm{~s}^{-1}\right)\end{array}$ & $e$ & $\begin{array}{c}\omega \\
\text { (degrees) }\end{array}$ & $\begin{array}{c}T_{0} \\
(\mathrm{JD}-2450000)\end{array}$ & $\begin{array}{l}M \sin i \\
\left(\mathrm{M}_{\mathrm{JUP}}\right)\end{array}$ & $\begin{array}{c}a \\
(\mathrm{AU})\end{array}$ & $\mathrm{N}_{o b s}$ & $\begin{array}{c}\text { RMS } \\
\left(\mathrm{m} \mathrm{s}^{-1}\right)\end{array}$ \\
\hline 111232 & $1118(30)$ & $162(20)$ & $0.19(0.10)$ & $82(15)$ & 3501 (35) & 6.7 & 1.9 & 15 & 4.70 \\
\hline 48265 & $762(50)$ & $29(6)$ & $0.24(0.10)$ & $289(50)$ & 2892 (100) & 1.2 & 1.6 & 17 & 5.14 \\
\hline 28185 & $379.0(2)$ & $163.5(3)$ & $0.05(0.03)$ & $44(2)$ & $4230.5(2)$ & 6.7 & 1.1 & 15 & 7.33 \\
\hline 143361 & $1086(90)$ & $63(20)$ & $0.18(0.10)$ & $187(32)$ & 3439 (190) & 3.0 & 2.0 & 12 & 3.88 \\
\hline 43848 & $2371(840)$ & $544(200)$ & $0.69(0.12)$ & $229(9)$ & 3227 (65) & 25 & 3.4 & 10 & 7.16 \\
\hline
\end{tabular}


Table 3. Velocities for HD 111232

\begin{tabular}{rrr}
\hline \hline JD & $\begin{array}{r}\text { RV } \\
(-2450000)\end{array}$ & $\begin{array}{r}\text { error } \\
\left(\mathrm{m} \mathrm{s}^{-1}\right)\end{array}$ \\
\hline 863.4875 & -28.5 & 7.5 \\
1042.7591 & 90.0 & 7.8 \\
1127.7398 & 123.6 & 3.3 \\
1455.8151 & 108.0 & 3.2 \\
1542.5330 & -6.8 & 3.5 \\
1756.8718 & -128.6 & 2.8 \\
1757.8713 & -129.6 & 2.7 \\
1774.8630 & -137.6 & 3.3 \\
1775.8698 & -130.2 & 3.7 \\
1783.8728 & -128.7 & 2.8 \\
1785.8745 & -125.2 & 2.8 \\
1811.8014 & -116.2 & 2.9 \\
1872.6422 & -101.0 & 2.9 \\
1896.5819 & -80.0 & 3.5 \\
2189.8023 & 89.6 & 3.0 \\
\hline
\end{tabular}


Table 4. Velocities for HD 48265

\begin{tabular}{rrr}
\hline \hline $\begin{array}{r}\text { JD } \\
(-2450000)\end{array}$ & $\begin{array}{r}\text { RV } \\
\left(\mathrm{m} \mathrm{s}^{-1}\right)\end{array}$ & $\begin{array}{r}\text { error } \\
\left(\mathrm{m} \mathrm{s}^{-1}\right)\end{array}$ \\
\hline 920.8629 & 20.6 & 6.9 \\
1431.6073 & -30.2 & 2.5 \\
1455.5573 & -35.0 & 2.6 \\
1685.8138 & 15.0 & 2.9 \\
1774.6744 & 25.0 & 3.3 \\
1775.6763 & 12.4 & 5.2 \\
1784.6887 & 25.4 & 2.6 \\
1811.5943 & 24.3 & 2.5 \\
1987.9168 & -7.7 & 2.9 \\
2078.7826 & -17.9 & 3.0 \\
2081.7133 & -23.4 & 2.8 \\
2137.6436 & -20.7 & 2.5 \\
2138.6670 & -28.7 & 2.6 \\
2189.5669 & -29.5 & 4.4 \\
2483.6148 & 28.5 & 3.0 \\
2501.6725 & 21.5 & 2.4 \\
2522.6219 & 25.4 & 2.7 \\
\hline
\end{tabular}


Table 5. Velocities for HD 28185

\begin{tabular}{rrr}
\hline \hline $\begin{array}{r}\mathrm{JD} \\
(-2450000)\end{array}$ & $\begin{array}{r}\mathrm{RV} \\
\left(\mathrm{m} \mathrm{s}^{-1}\right)\end{array}$ & $\begin{array}{r}\text { error } \\
\left(\mathrm{m} \mathrm{s}^{-1}\right)\end{array}$ \\
\hline 627.6637 & 120.0 & 5.2 \\
663.5918 & 158.0 & 6.3 \\
918.7297 & -92.5 & 4.9 \\
920.7447 & -87.8 & 4.7 \\
981.7281 & 72.2 & 5.5 \\
1306.8369 & -54.6 & 5.5 \\
1307.8161 & -72.9 & 5.5 \\
1431.5270 & 167.8 & 3.9 \\
1455.4900 & 147.1 & 4.1 \\
1656.8504 & -123.6 & 4.2 \\
1657.8599 & -121.7 & 4.2 \\
1685.7325 & -60.5 & 4.1 \\
1757.6165 & 104.4 & 4.1 \\
2077.6809 & -34.2 & 3.9 \\
2079.7824 & -40.0 & 3.8 \\
\hline
\end{tabular}


Table 6. Velocities for HD 143361

\begin{tabular}{rrr}
\hline \hline $\begin{array}{r}\text { JD } \\
(-2450000)\end{array}$ & $\begin{array}{r}\text { RV } \\
\left(\mathrm{m} \mathrm{s}^{-1}\right)\end{array}$ & $\begin{array}{r}\text { error } \\
\left(\mathrm{m} \mathrm{s}^{-1}\right)\end{array}$ \\
\hline 864.5793 & 62.1 & 6.8 \\
1130.8371 & 19.5 & 2.4 \\
1872.7370 & 53.2 & 2.5 \\
1987.5051 & 62.2 & 3.0 \\
1988.4946 & 51.3 & 2.6 \\
2190.8055 & 25.7 & 3.2 \\
2217.8473 & 20.0 & 3.0 \\
2277.6582 & -0.4 & 3.2 \\
2299.5595 & -8.3 & 2.6 \\
2300.5804 & -7.7 & 2.2 \\
2339.5005 & -23.6 & 3.2 \\
2501.8720 & -68.8 & 2.9 \\
\hline
\end{tabular}


Table 7. Velocities for HD 43848

\begin{tabular}{rrr}
\hline \hline $\begin{array}{r}\text { JD } \\
(-2450000)\end{array}$ & $\begin{array}{r}\text { RV } \\
\left(\mathrm{m} \mathrm{s}^{-1}\right)\end{array}$ & $\begin{array}{r}\text { error } \\
\left(\mathrm{m} \mathrm{s}^{-1}\right)\end{array}$ \\
\hline 626.7557 & 124.1 & 5.8 \\
920.8278 & -90.8 & 6.0 \\
978.7597 & -173.4 & 7.2 \\
1431.5773 & 498.7 & 3.5 \\
1455.5289 & 521.2 & 3.7 \\
1774.6672 & 479.7 & 4.2 \\
1775.6690 & 486.9 & 7.1 \\
1783.6628 & 483.0 & 3.6 \\
1810.5720 & 486.9 & 3.5 \\
2136.6677 & 406.5 & 3.7 \\
\hline
\end{tabular}




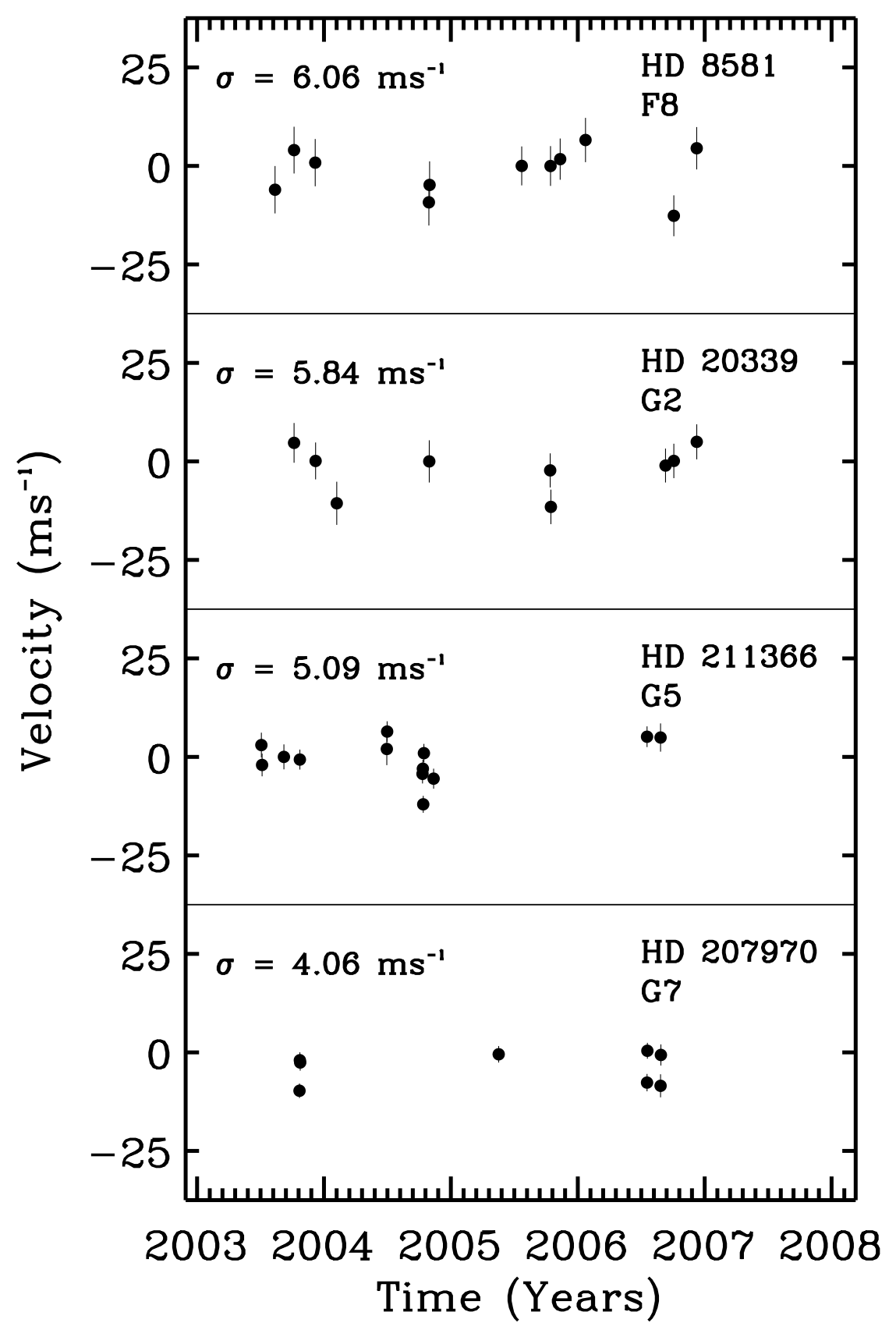

Fig. 1.- Four MIKE stable stars with spectral types ranging from late $\mathrm{F}$ to mid G. 


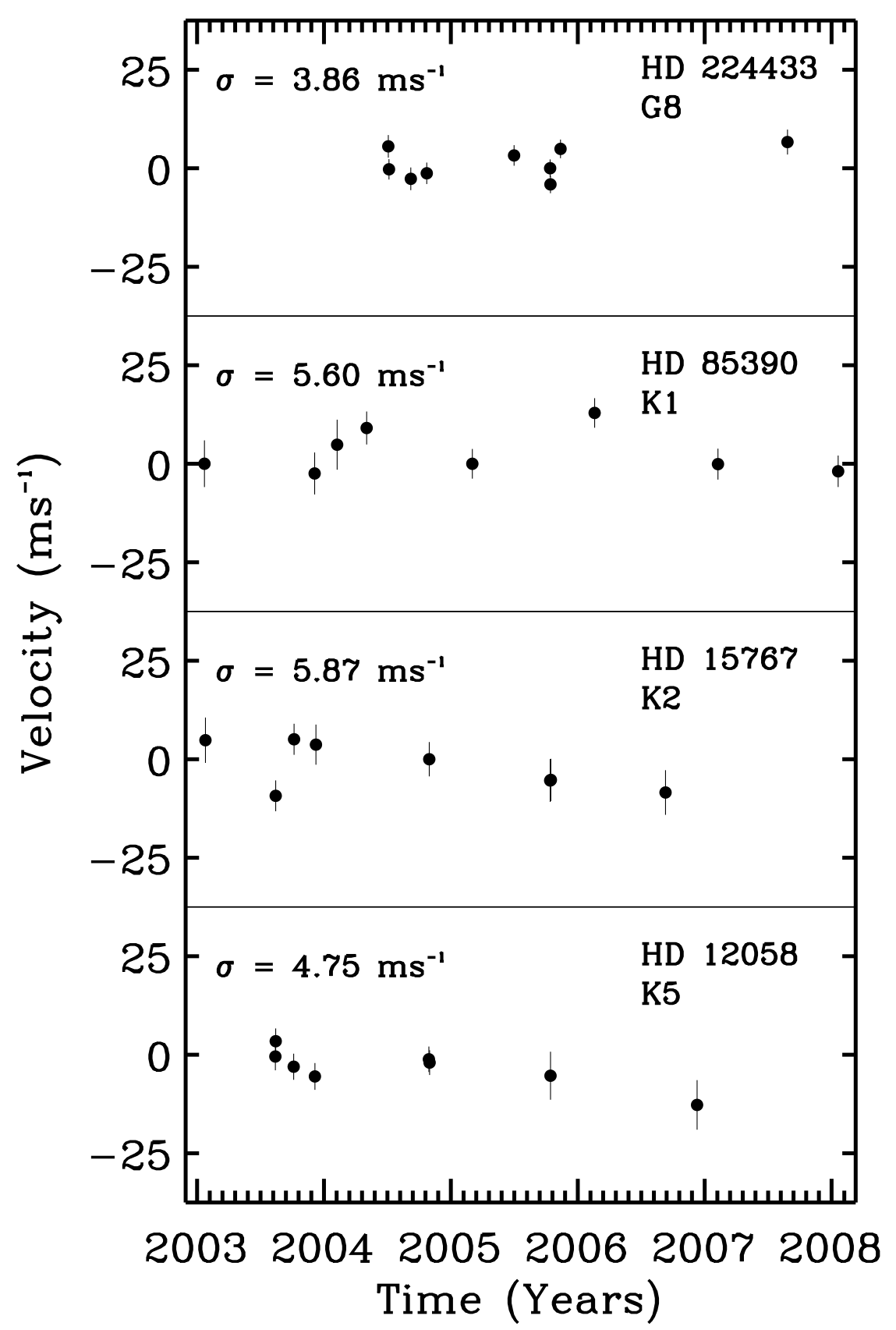

Fig. 2.- Four MIKE stable stars with spectral types ranging from late G to mid K. 


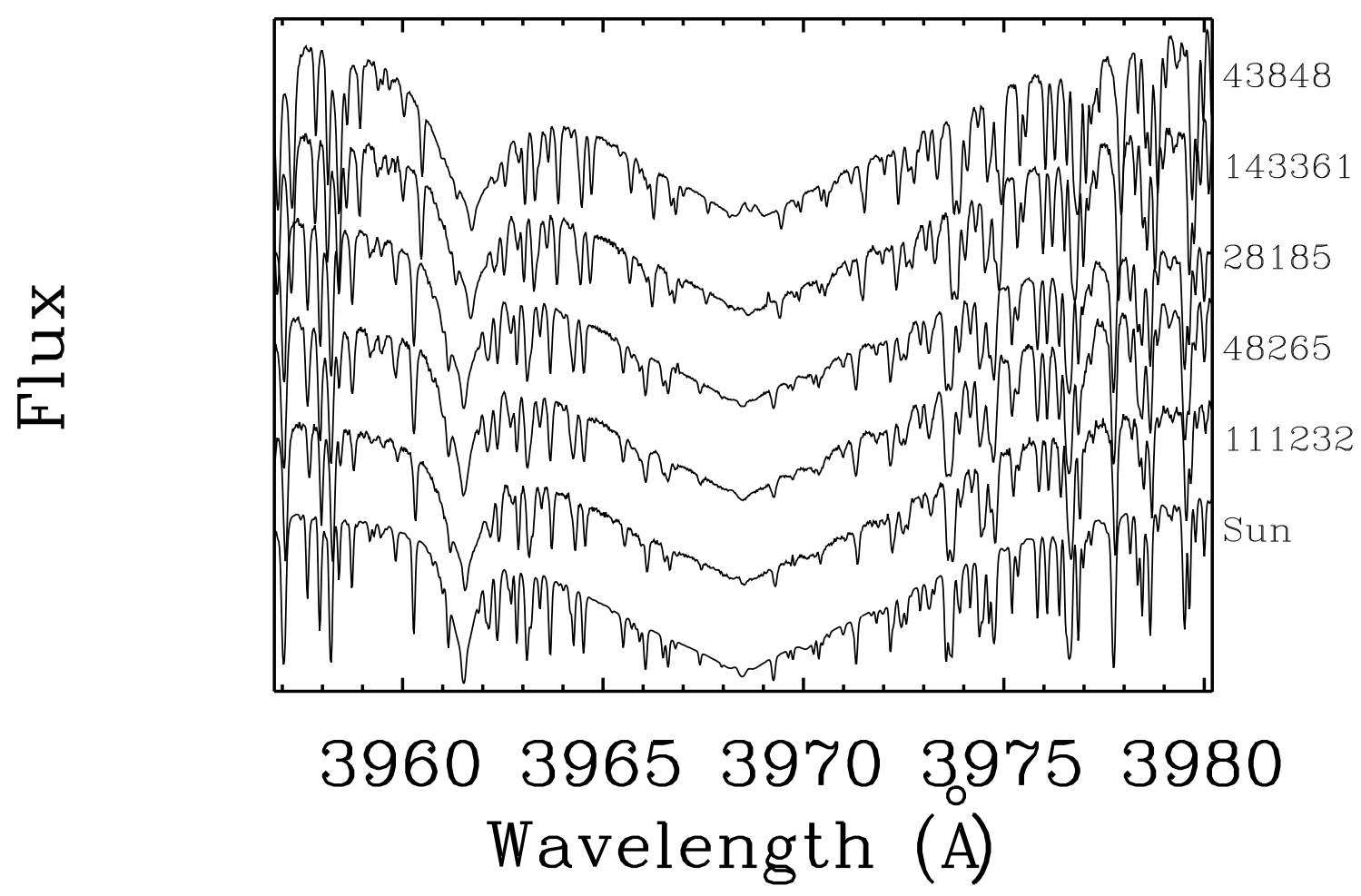

Fig. 3.- Ca II $\mathrm{H}$ line cores for the five target $\mathrm{G}$ dwarfs in ascending order of $B-V$. The HD catalog number of each star is shown along the right edge. The Sun is shown for comparison. The $\mathrm{R}_{\mathrm{HK}}$ values derived from the $\mathrm{H} \& \mathrm{~K}$ lines are similar to the Sun, indicating rotation periods of $25 \mathrm{~d}$ or longer and photospheric Doppler "jitter" of $3 \mathrm{~m} \mathrm{~s}^{-1}$ or less. 


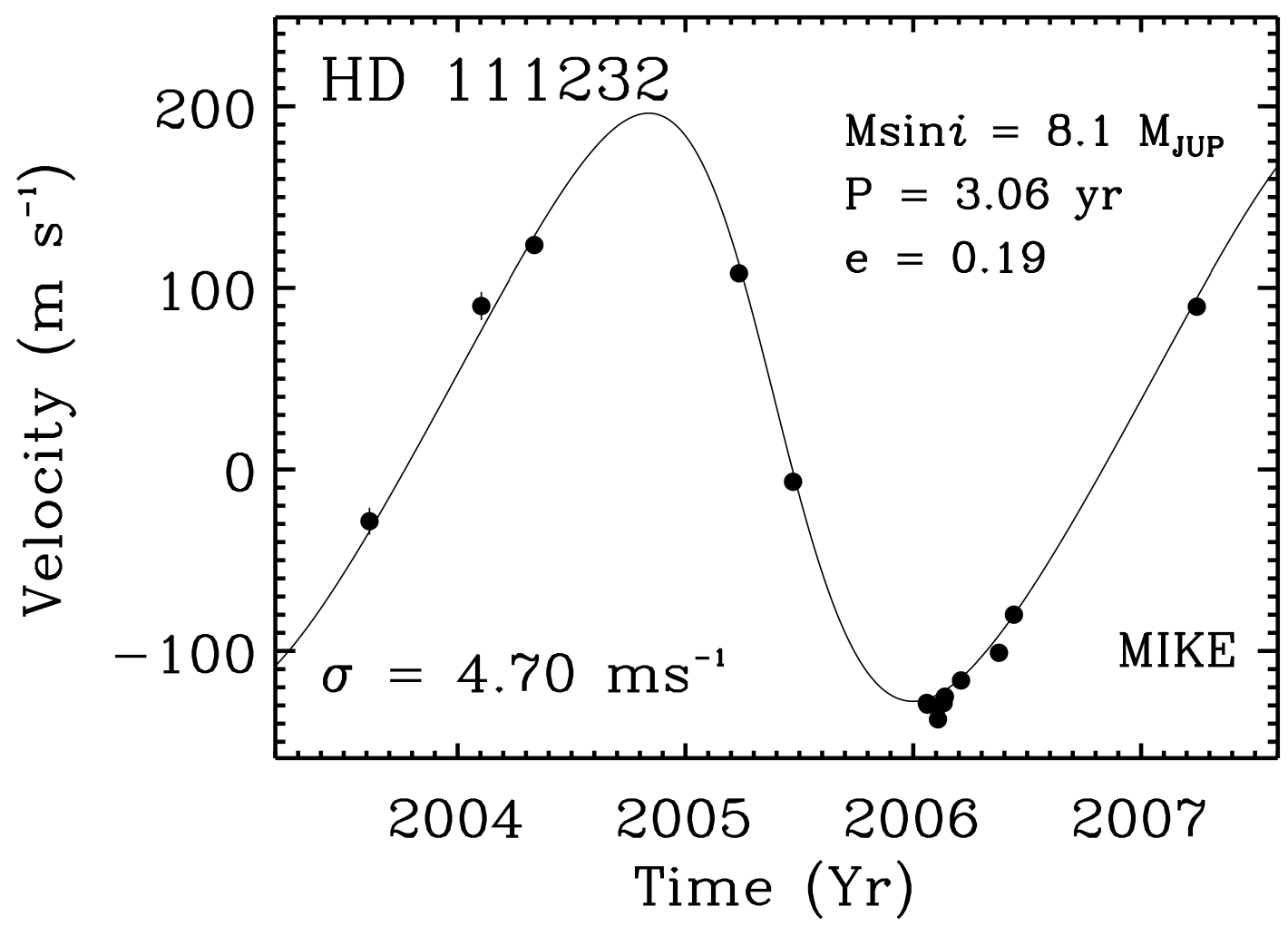

Fig. 4. - Doppler velocities for HD 111232 (G5 V). The solid line is a Keplerian orbital fit with a period of 1118 days, a semi-amplitude of $172 \mathrm{~m} \mathrm{~s}^{-1}$, and an eccentricity of 0.19 , yielding a minimum $(M \sin i)$ of $8.1 \mathrm{M}_{\text {JUP }}$ for the companion. The RMS of the Keplerian fit is $4.70 \mathrm{~m} \mathrm{~s}^{-1}$. 


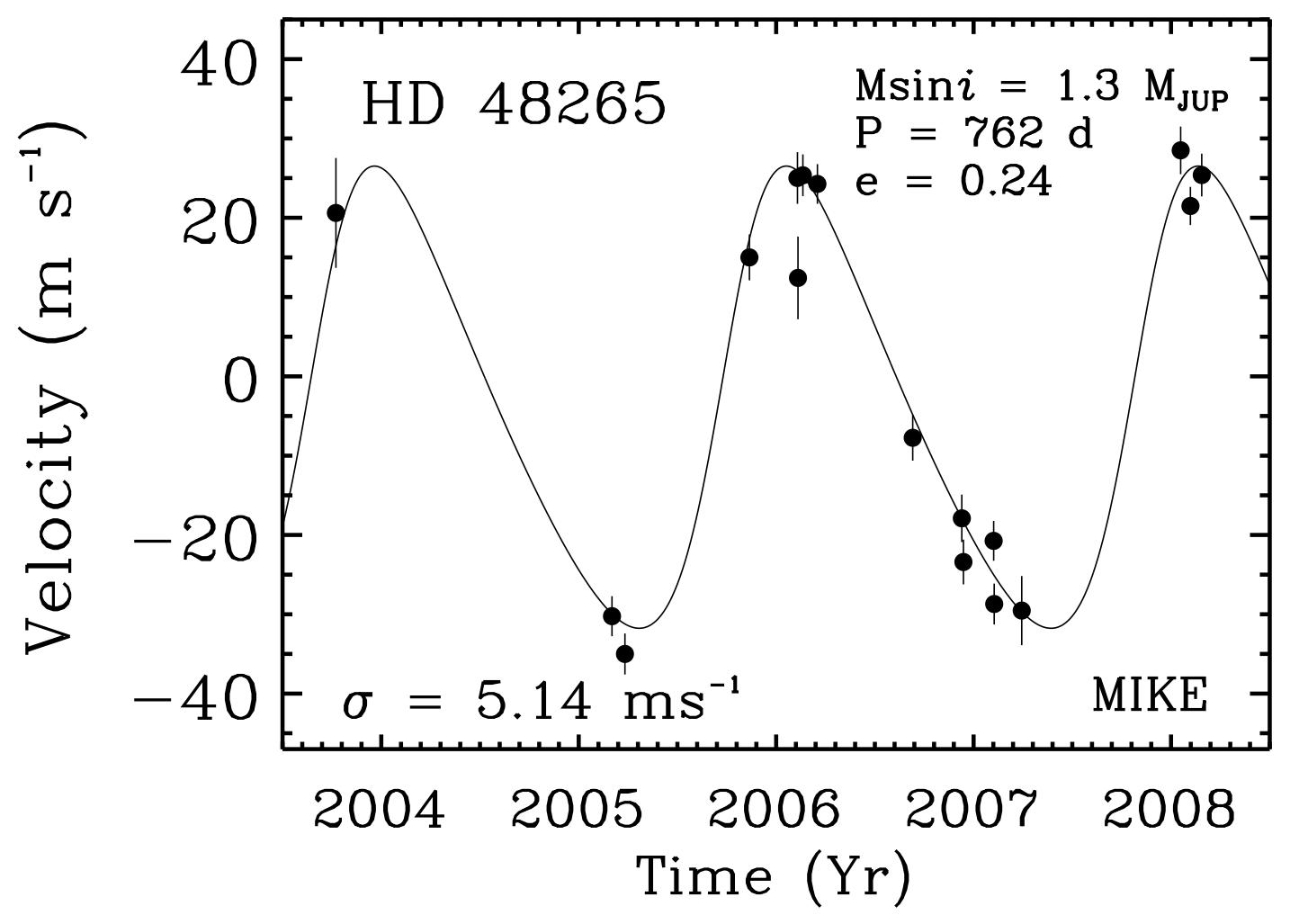

Fig. 5.- Doppler velocities for HD 48265 (G5 V). The solid line is a Keplerian orbital fit with a period of 762 days, a semi-amplitude of $29 \mathrm{~m} \mathrm{~s}^{-1}$, and an eccentricity of 0.24 , yielding a minimum $(M \sin i)$ of $1.3 \mathrm{M}_{\mathrm{JUP}}$ for the companion. The RMS of the Keplerian fit is 5.14 $\mathrm{m} \mathrm{s}^{-1}$. 


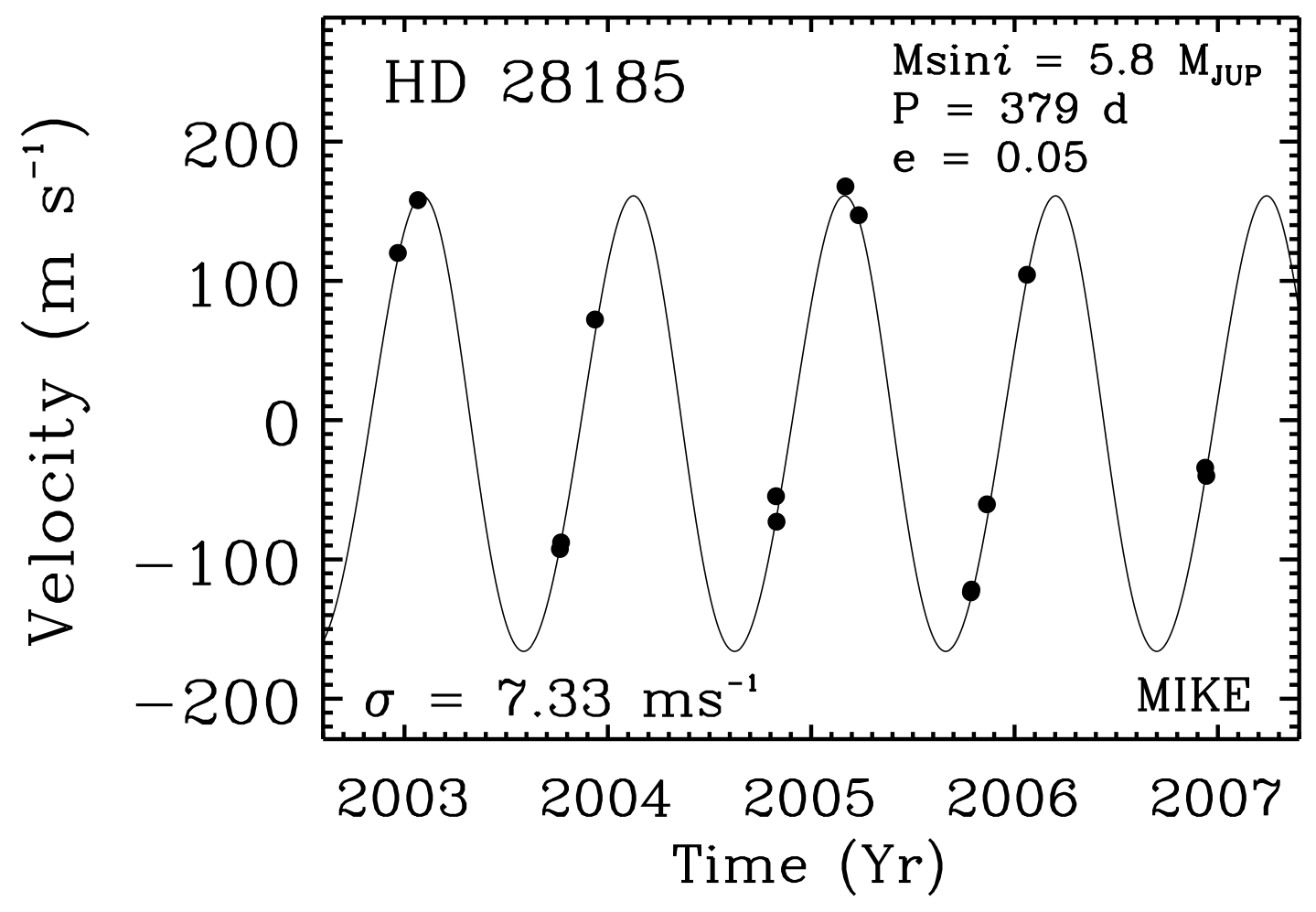

Fig. 6.- Doppler velocities for HD 28185 (G0 V). The solid line is a Keplerian orbital fit with a period of 379 days, a semi-amplitude of $163 \mathrm{~m} \mathrm{~s}^{-1}$, and an eccentricity of 0.05 , yielding a minimum $(M \sin i)$ of $5.8 \mathrm{M}_{\text {JUP }}$ for the companion. The RMS of the Keplerian fit is $7.33 \mathrm{~m} \mathrm{~s}^{-1}$. 


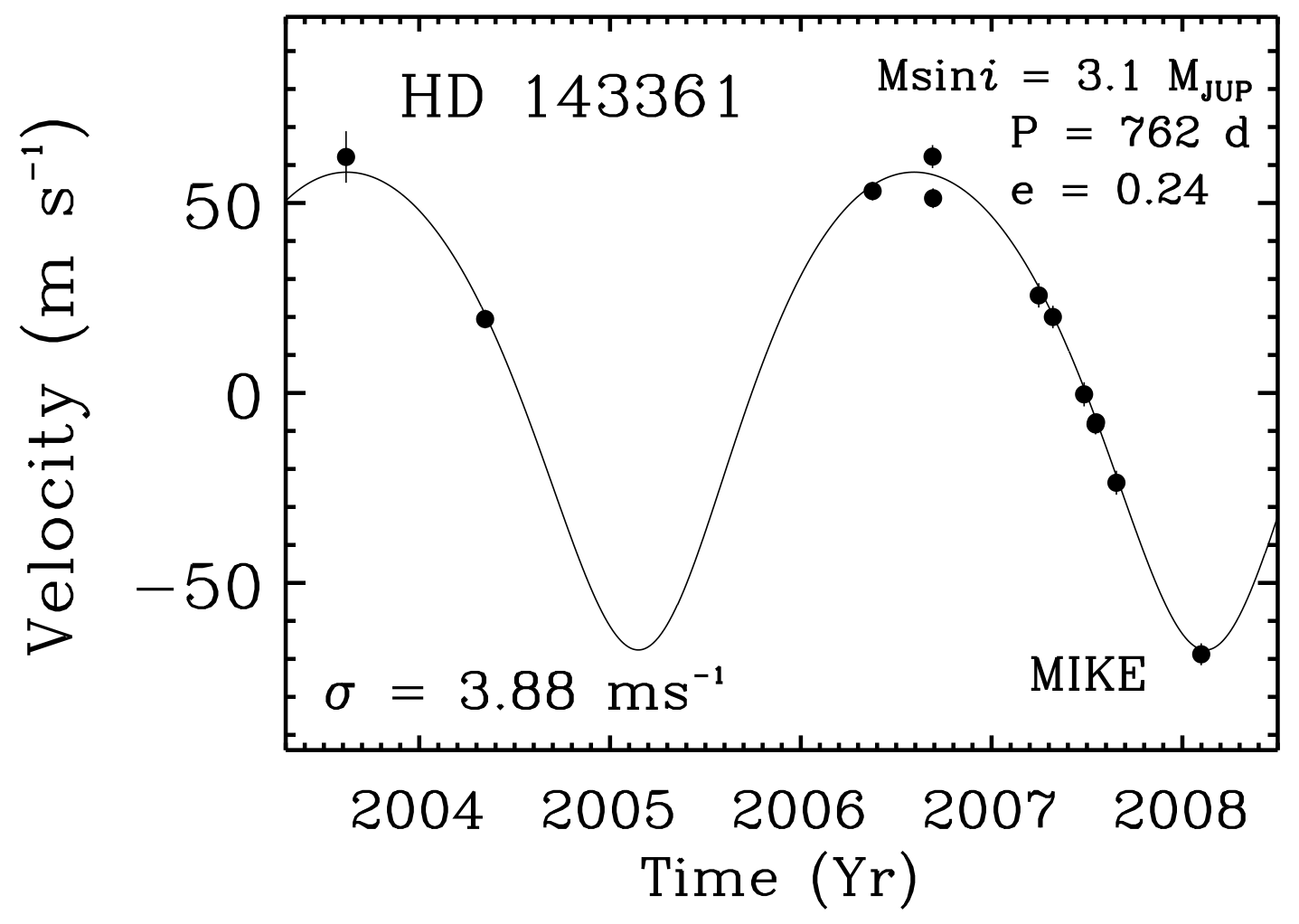

Fig. 7.- Doppler velocities for HD 143361 (G0 V). The solid line is a Keplerian orbital fit with a period of 1086 days, a semi-amplitude of $63 \mathrm{~m} \mathrm{~s}^{-1}$, and an eccentricity of 0.18 , yielding a minimum $(M \sin i)$ of $3.1 \mathrm{M}_{\mathrm{JUP}}$ for the companion. The RMS of the Keplerian fit is $3.88 \mathrm{~m} \mathrm{~s}^{-1}$. 


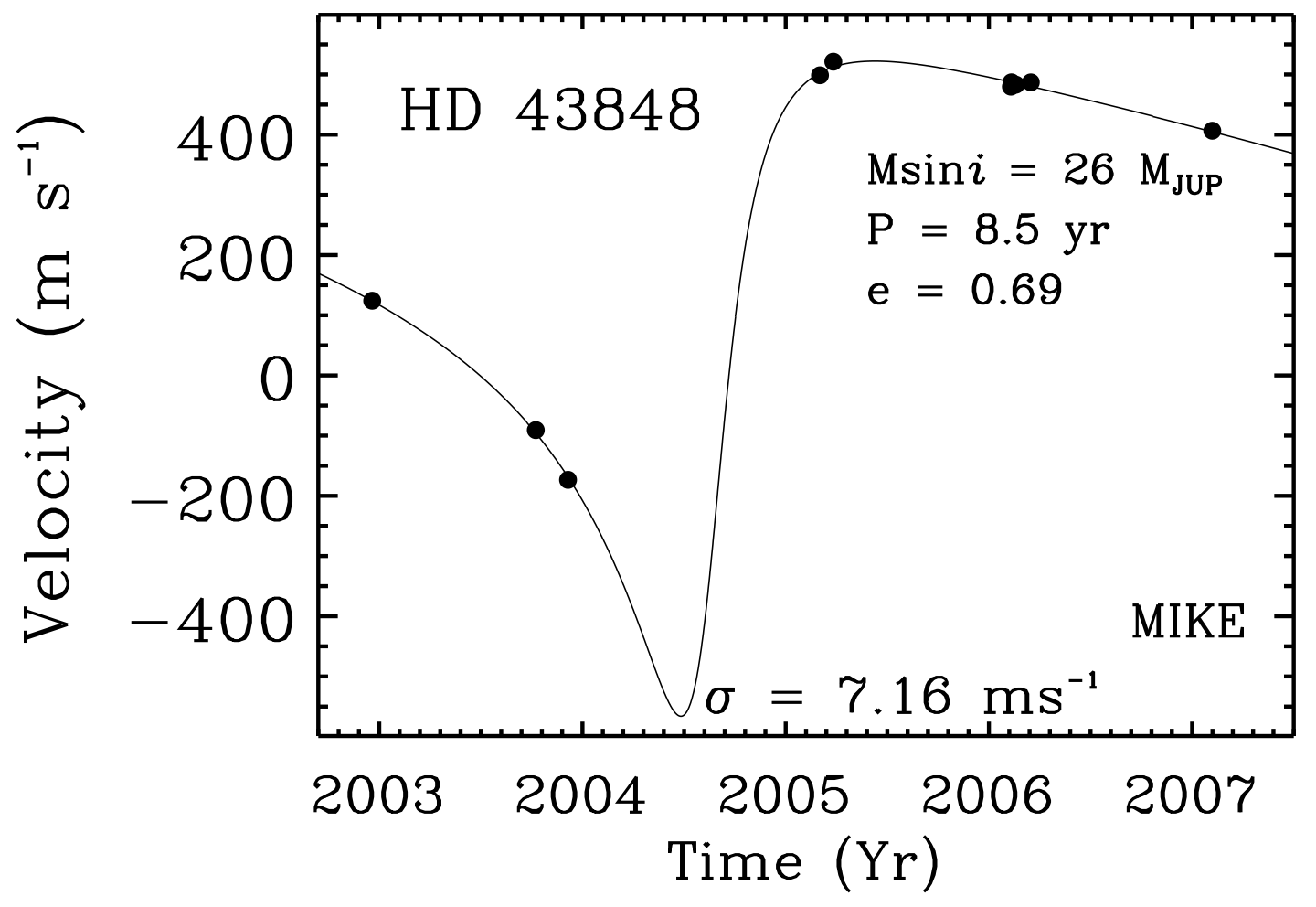

Fig. 8.- Doppler velocities for HD 43848 (G2 V). The solid line is a Keplerian orbital fit with a period of 2371 days, a semi-amplitude of $544 \mathrm{~m} \mathrm{~s}^{-1}$, and an eccentricity of 0.69 , yielding a minimum $(M \sin i)$ of $26 \mathrm{M}_{\mathrm{JUP}}$ for the companion. The RMS of the Keplerian fit is $7.16 \mathrm{~m} \mathrm{~s}^{-1}$. 


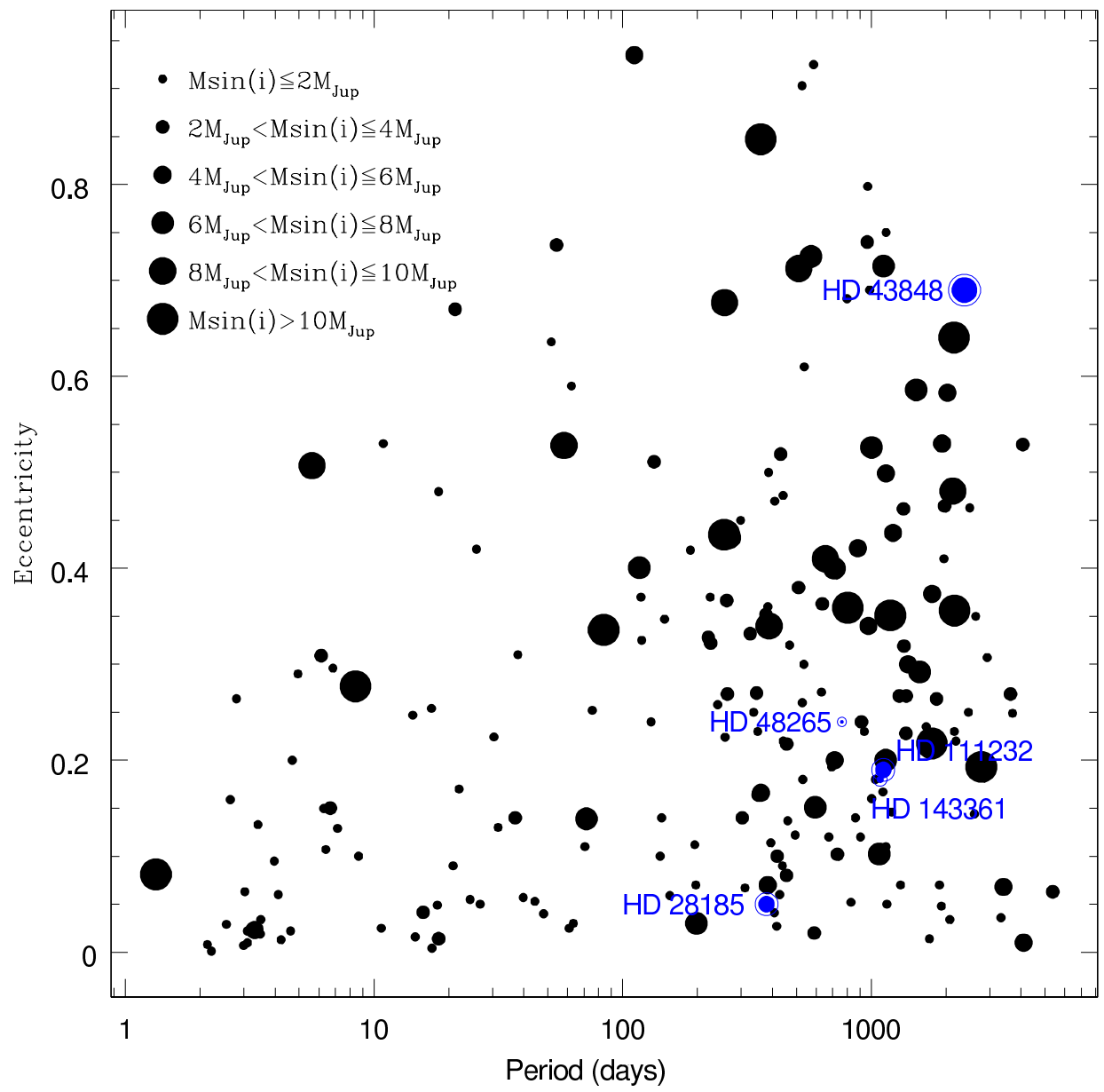

Fig. 9.- Orbital eccentricity versus period for known extrasolar planetary hosts. The circle sizes are proportional to planet mass as indicated. The objects studied here are shown with large full circles. Note the extreme position of HD 43848, as a very massive and eccentric object. 


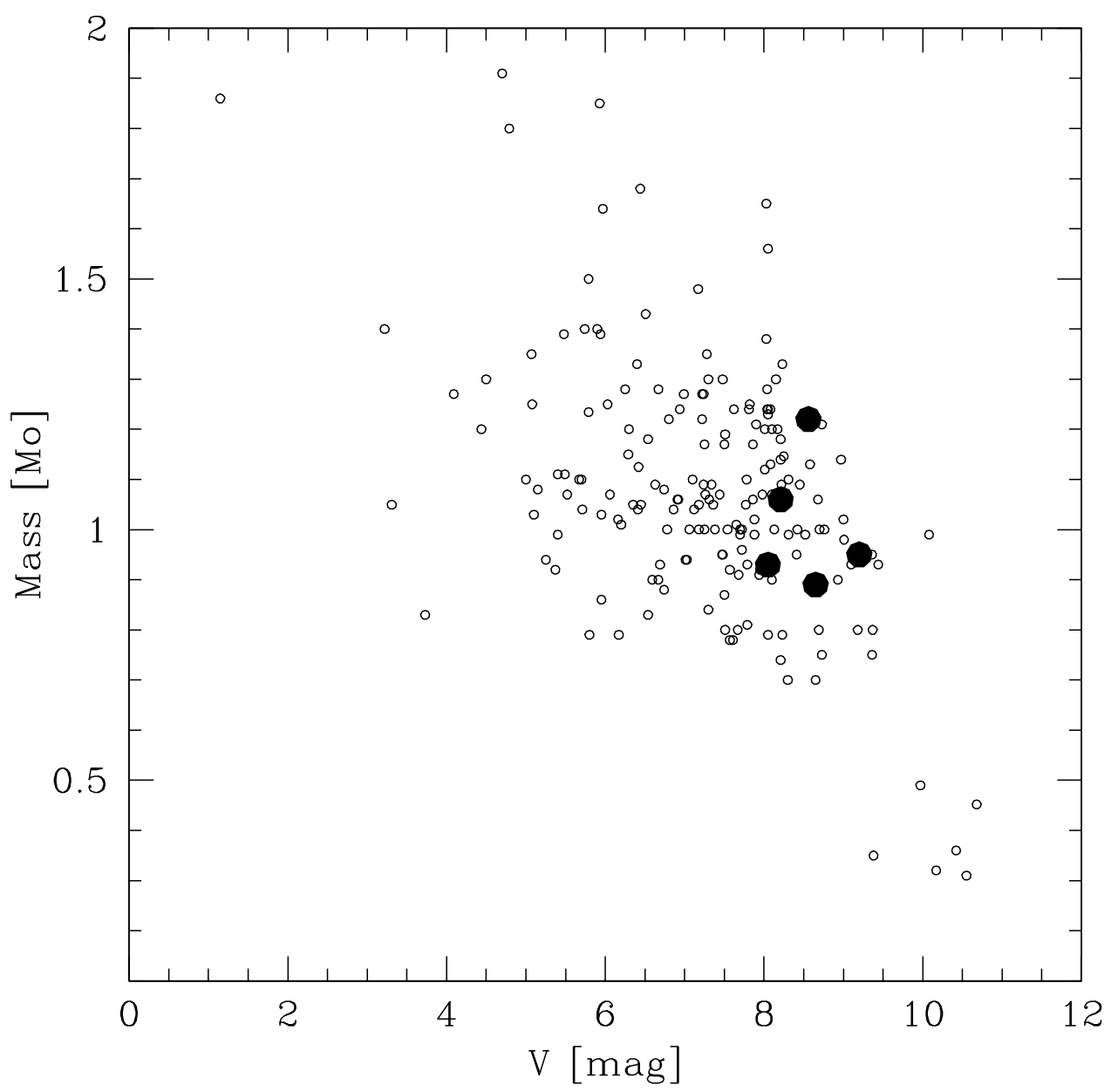

Fig. 10.- Star mass versus apparent $V$ magnitude for known extrasolar planetary hosts (small circles). The new planets from our survey (three from this work plus two from LopezMoralez et al. 2008) are shown with large full circles. These first Magellan discoveries are among the faintest targets surveyed for planets, probing deeper in the Solar Neighborhood. The two previous CORALIE detections are included among the small circles. 